\title{
Corrigendum
}

\section{Corrigendum to "Quality of Life and Sexual Health in the Aging of PCa Survivors"}

\author{
Mauro Gacci, ${ }^{1}$ Elisabetta Baldi, ${ }^{2}$ Lara Tamburrino, ${ }^{2}$ Beatrice Detti, ${ }^{3}$ Lorenzo Livi, ${ }^{3}$ \\ Cosimo De Nunzio, ${ }^{4}$ Andrea Tubaro, ${ }^{4}$ Stavros Gravas, ${ }^{5}$ Marco Carini, ${ }^{1}$ and Sergio Serni ${ }^{1}$ \\ ${ }^{1}$ Department of Urology, University of Florence, Careggi Hospital, Viale Gramsci 7, 50121 Florence, Italy \\ ${ }^{2}$ Department of Experimental and Clinical Biomedical Sciences, Section of Clinical Pathophysiology, University of Florence, Italy \\ ${ }^{3}$ Radiotherapy, University Hospital Careggi, University of Florence, Italy \\ ${ }^{4}$ Department of Urology, Sant'Andrea Hospital, University "La Sapienza", Rome, Italy \\ ${ }^{5}$ Department of Urology, University Hospital of Larissa, Larissa, Greece
}

Correspondence should be addressed to Mauro Gacci; maurogacci@yahoo.it

Received 16 September 2014; Accepted 19 October 2014

Copyright (C) 2015 Mauro Gacci et al. This is an open access article distributed under the Creative Commons Attribution License, which permits unrestricted use, distribution, and reproduction in any medium, provided the original work is properly cited.

In the paper "Quality of Life and Sexual Health in the Aging of PCa Survivors" there are 2 mistakes at page 9, in the paragraph "5.4. Androgen Deprivation Therapy (ADT)": "[162]" has to be replaced by the words "with LHRH agonists," while "[162]" has to be shifted at the end of the second and third sentences (lines 6 and 9, resp.). 


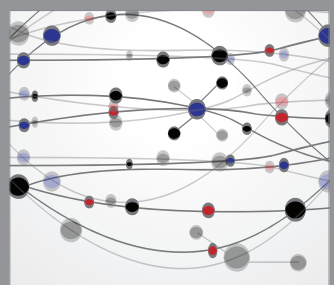

The Scientific World Journal
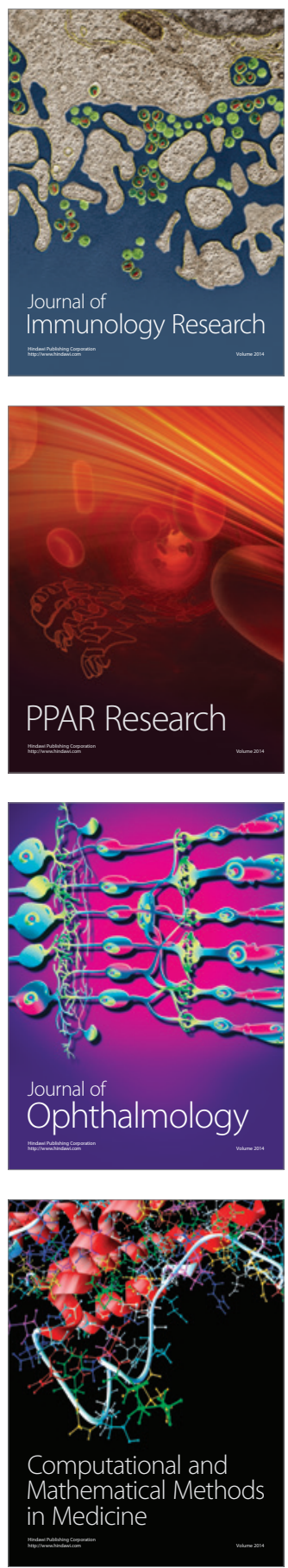

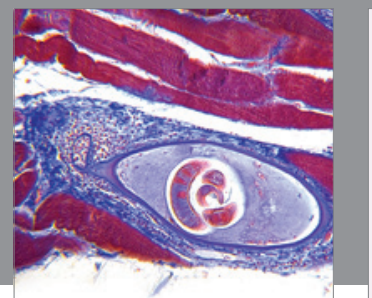

Gastroenterology

Research and Practice
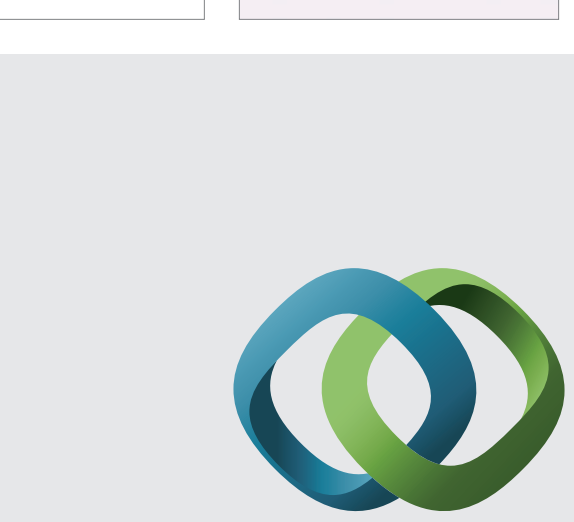

\section{Hindawi}

Submit your manuscripts at

http://www.hindawi.com
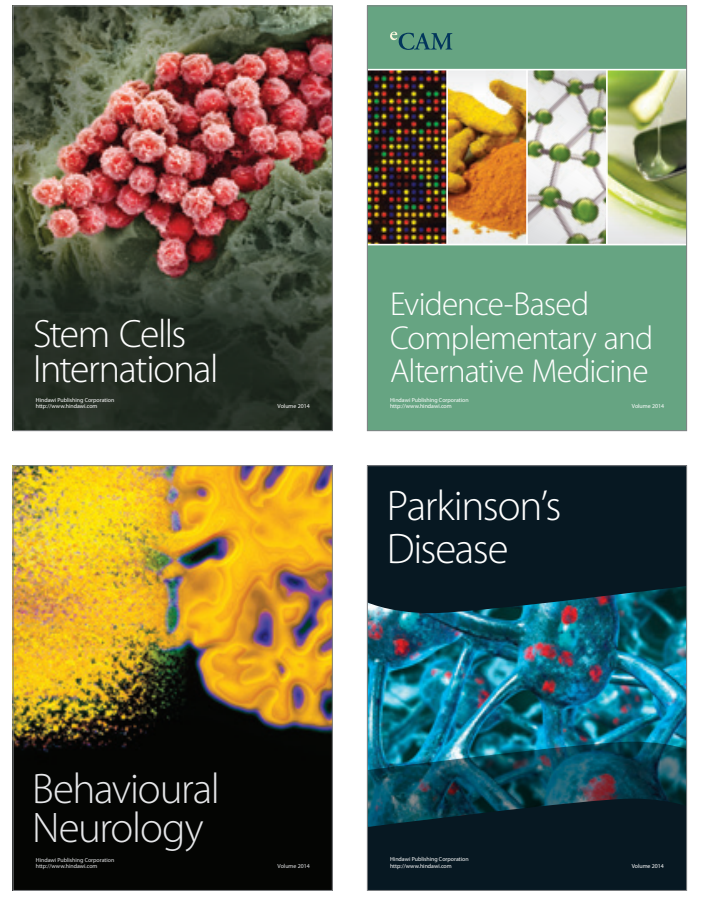
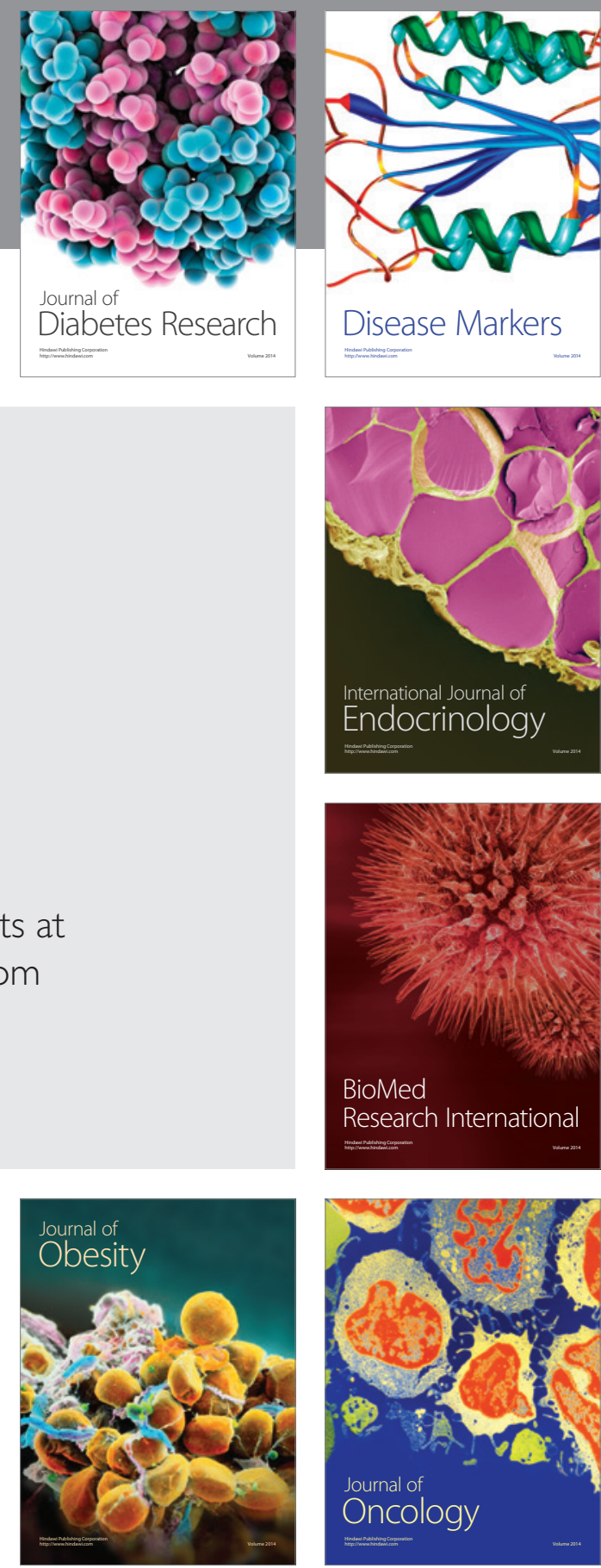

Disease Markers
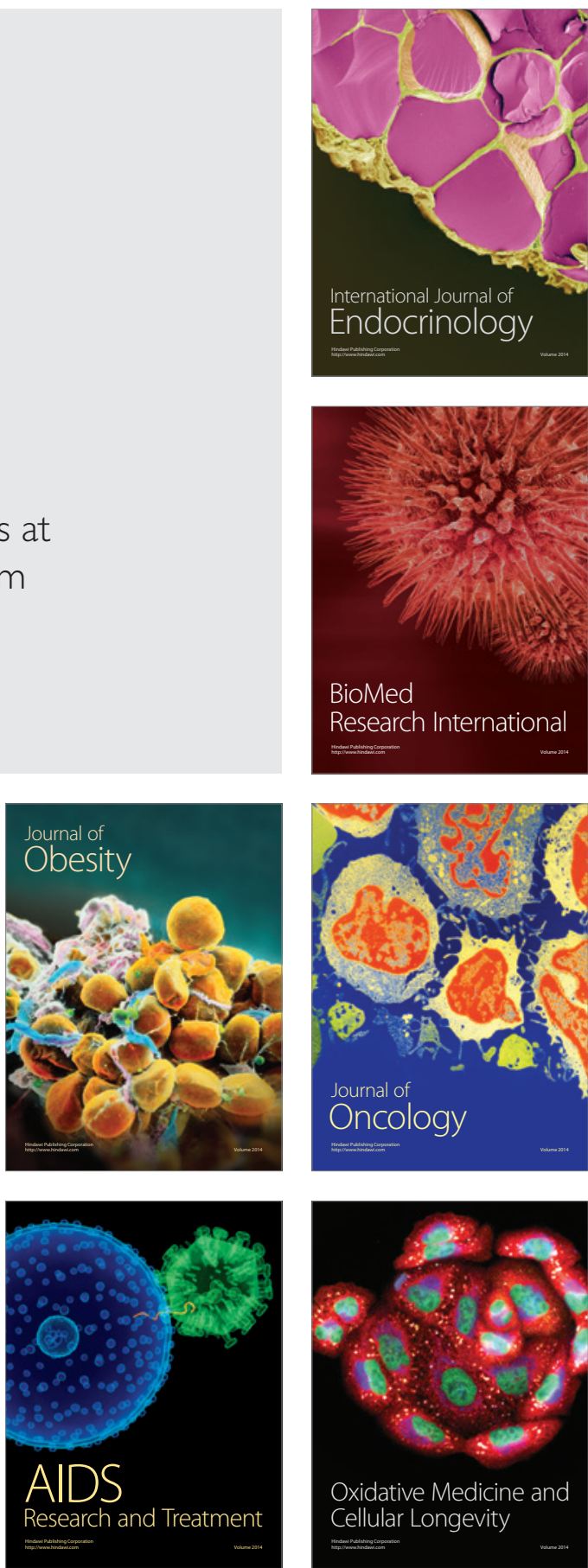